\title{
Assessment of the Consistency of Tetabulin Injection to the Patients with an Open Fracture Referred to the Khatamolanbia Hospital, Zahedan in 2017 with the National Guidelines
}

\author{
Mohammad Sedaghat $^{1}$, Alireza Dashipour ${ }^{2} \&$ Mahtab Masood $^{3}$ \\ ${ }^{1}$ Emergency Medicine, Department of Medical Emergencies, School of Medicine, Khatam Al Anbiya Hospital, \\ Zahedan University of Medical Sciences, Iran \\ ${ }^{2}$ Nutritional Sciences \& Food Technology, Department of Nutrition, School of Medicine, Cellular and \\ Molecular Research Center, Resistant Tuberculosis Institute, Zahedan University of Medical Sciences, Iran \\ ${ }^{3}$ Emergency Medicine, Zahedan University of Medical Sciences, Zahedan, Iran \\ Correspondence: Mohammad Sedaghat, Emergency Medicine, Department of Medical Emergencies, School of \\ Medicine, Khatam Al Anbiya Hospital, Zahedan University of Medical Sciences, Iran.
}

Received: May 7, 2020

doi:10.5539/jmbr.v10n1p148
Accepted: July 19, 2020

Online Published: November 30, 2020

URL: https://doi.org/10.5539/jmbr.v10n1p148

\begin{abstract}
Background and Goal: Open fractures are at risk of infection with Clostridium tetani and severe traumatic infections. Tetabulin injection is strongly recommended for the patients with an open fracture and severe wounds. The goal of this study is to assess the consistency of tetabulin injection to the patients with an open fracture referred to the Khatamolanbia hospital in Zahedan in 2017 with the national guidelines.

Materials and Methods: This study is a cross-sectional descriptive study. 300 patients with an open fracture referred to the ER of the Khatamolanbia Hospital in Zahedan in 2017 were selected as the sample. Their fracture type and severity were assessed. The data were classified in the tables and statistically analyzed using Chi-square, pared t-test, Pearson correlation, and regression in SPSS 26.
\end{abstract}

Findings: Among 300 patients, 275 patients (91.7\%) were male and 25 patients (8.3\%) were female. The most frequent age range was 20 to 30 years old $(31.7 \%)$, and the least frequent ones were 5 to 10 years old $(10 \%)$ and more than 50 years old $(11.6 \%)$. The results showed that gender has no significant effect on the predictability of the need of tetabulin injection for the patients with open fractures $(\mathrm{P}=0.780)$. However, age has a significant positive effect on the predictability of the need of tetabulin injection for the patients with open fractures; as the age increases, the need for tetabulin injection also increases, and it must be injected in the 50 years and older patients $(\mathrm{P}=0.05)$.

Conclusion: The results showed that age was effective on the decrease of the serum level of anti-tetanus antibody, however, gender had no significant effect on it. Therefore, it is concluded that tetabulin injection for open fractures is consistent with the national guideline.

Keywords: Tetabulin, Open Fracture, Tetanus Toxin, Trauma

\section{Introduction}

Accident is the most frequent cause of an open fracture and severe wound in Iran (Jafari, Abolhasani, Naghavi, Pourmalek, \& Moradi, 2009). The wounds due to accident are one of the most common complication in the ERs. Open fractures and wounds are at risk of infection with bacteria such as Clostridium tetani. Tetanus is a dangerous disease which is caused by the entrance of Clostridium tetani (tetanus) into the wound (Mokhayeri et al., 2016). This bacterium produces a dangerous toxin in the wound which harms the nervous system and leads into loose paralysis or spasm of the muscles (especially the paralysis of the respiratory muscles). Severe muscular spasm due to tetanus leads to the serious health complications such as respiratory problems due to the spasm of the vocal cords (laryngospasm) and muscles that control respiration, pneumonia (lung infection), brain injuries due to the lack of oxygen, cardiac arrhythmia, bone and spinal fracture due to muscular spasm, and seizure (Qadir \& Komal, 2019). Due to the high rate of mortality of tetanus, the assessment of the immune condition of the patients and making decision about the need for the injection of tetabulin necessary. This 
assessment is performed in different ways, such as doctor's judgment about the condition of the infected wound, the time of the previous vaccination based on the recommended protocols in the reference books and world health organization (WHO), rapid diagnostic methods, and ELISA test (Dong, Masuyer, \& Stenmark, 2019). Therefore, since the treatment of tetanus is very difficult, prevention by vaccination is the best thing to do. The most important method to prevent tetanus infection in the open fractures and wounds, especially in those who have unknown or incomplete (less than 3 times) tetanus vaccination history, is Tetanus Immune Globulin (TIG) injection under the brand name tetabulin (Finkelstein, Teisch, Allen, \& Ruiz, 2017). Tetabulin is a hyper immune sterile solution that creates passive immunity against tetanus. In fact, this treatment is a type of immune transmission against the tetanus toxin in a passive manner (O'Dwyer, 2017). Therefore, in patients with an open fracture and severe wounds, tetabulin injection is strongly recommended to prevent tetanus infection. The mechanism of tetanus immunoglobulin is that the immunoglobulins attach to the tetanus toxoids and prevent the normal attachment of them to the body tissues. Therefore, the immunoglobulins inhibit the entrance of the toxoids into the nervous system and prevent the painful muscular spasms and brain function disorders. Clostridium tetani is destroyed by the antibiotic produced by the host body. Furthermore, the immunoglobulin attached to the bacterial toxoid destroys the bacteria by inducing phagocytosis in the immune system of the host (Graham \& Ambrosino, 2015).

Tetanus immunoglobulin is a product produced by the human plasma, and it consists of the antibodies against the tetanus toxoids. Tetabulin is generally used to prevent tetanus in the patients with severe wounds. Tetanus toxin produced by Clostridium tetani leads to severe spasm, and in $30-40 \%$ of the cases, it leads to spinal fracture and death. Tetabulin injection is used for protection and prevention against the tetanus toxin in the unimmunized individuals. Also, tetabulin injection is necessary while there is an open fracture in the patients with immunodeficiency and the individuals who hasn't been vaccinated with the anti-tetanus vaccine during the last 5 years. The recommended dose for these individuals is 250 I.U, and it is doubled (500 I.U.) for those whose weight is more than $90 \mathrm{Kgs}$ and the individuals who have an open fracture for more than 24 hours (Yen, Murray, Zipprich, Winter, \& Harriman, 2015). Tetanus immunoglobulin is contraindicated for those with thrombocytopenia, coagulation disorders, and hypersensitivity to Gamma globulin and immunoglobulin A. The side effects of tetanus immunoglobulin include nephrotic syndrome, anaphylaxis, nausea, vomiting, hives, fever, chest pain, lethargy, pain in the injection site, and angioneurotic edema. Therefore, the injection of live viral vaccines is contraindicated up to 3 months after tetanus immunoglobulin injection. Since tetanus immunoglobulin is made of human plasma, there is a possibility of the transmission of viral infection with the reactions such as wheezing, heavy feeling in the chest, fever, itching, severe cough, cyanosis, seizure, and edema in the face, lips, tongue, and throat. The side effects of this medicine have not yet been rejected, and there are no thorough human researches in this regard. Therefore, it is only prescribed when the doctor believes that its advantages surpass the possible disadvantages, and its use is necessary for the patient (Erdem \& Blankson, 2013).

In a study performed by Venturini et al., the immunity against tetanus toxin and the need for booster injection was assessed in the students and workers at risk of tetanus in a university in Italy. 95\% of 1433 individuals who were vaccinated during the past 10 years had high levels of blood antibody titer (more than 1.0). Also, those who got 5 doses of booster had very high and long-term immunity (more than $1.97 \mathrm{IU} / \mathrm{ml}$ ). This study suggested that if the individuals complete the vaccination period and then get 5 doses of boosters, they can be immune to tetanus for up to 20 years (Borella-Venturini et al., 2017). In study conducted by Nemati et al. (2015), the anti-tetanus antibody level in the patients with type II diabetes was assessed. The average antibody titer in these patients was lower than the normal population, and it was even lower in the female diabetic individuals in comparison to the male diabetic patients. They also found that there is a direct relationship between the decrease of the anti-tetanus antibody titer and the duration of diabetes, and it was lower in the patients with more than 5 years of diabetes. Therefore, they concluded that tetabulin injection in the diabetic patients with diabetic ulcers referred in the ERs is essential (Nemati et al., 2014). In a study administered by Khurana et al. (2014), they assessed the demographic data (gender and age) of the patients with tetanus and compared the treatment outcomes of intramuscular and spinal injection of tetabulin. They found that simultaneous injection of tetabulin (intramuscular and spinal) significantly increases the survival of the patients with tetanus (Narang, Khurana, Gomber, \& Choudhary, 2014).

Since there were not many related studies in this regard in Iran, the goal of this study was to assess the consistency of tetabulin injection to the patients with an open fracture referred to the Khatamolanbia hospital in Zahedan with the national guidelines. 


\section{Materials and Method}

The present study is a cross-sectional descriptive study. Its population is all the individuals with an open fracture. The inclusion criteria are having an open fracture and wound and no hypersensitivity to tetabulin, and the exclusion criterion is the lack of physical health of the patient that disables them from answering the questions properly. 300 individuals who had the inclusion criteria were selected using simple and available sampling from the patient cases in the hospital archive, and they were recorded in the researcher made checklists. This study is a prospective cross-sectional (descriptive-analytic) study to assess the consistency of tetabulin injection to the patients with an open fracture referred to the ER of the Khatamolanbia hospital in Zahedan in 2017 with the national guidelines. All the Helsinki principles are observed through this study, and it was approved by the ethics committee of the Zahedan University of Medical Sciences. First, the individuals with the inclusion/exclusion criteria were selected, then the tools used in this study were completely explained to them and their consent was taken.

Each patient was assessed according to the open fracture severity and tetabulin injection, and their data were recorded in the checklists and entered in the SPSS 26. Then, the results were statistically analyzed. The qualitative and quantitative variables were described by number (percent) and mean (standard deviation) respectively. To compare the mean score of the analytical experiences and psychological traumas, independent t-test and ANOVA were used. Finally, Pearson correlation and linear regression were used to assess the relationship between the analytical experiences and psychological traumas. The significance level of the tests was considered to be $0.05(\mathrm{P}=0.05)$.

\section{Findings}

Table 1. Patents' characteristics and the assessed variables (The frequency of the patients based on their gender, age, cause of fracture, the need for tetabulin injection, and the history of tetabulin injection)

\begin{tabular}{|c|c|c|}
\hline Frequency (percent) & Group & Variable \\
\hline $33(10.06 \%)$ & $5-10$ & \multirow{5}{*}{ Age } \\
\hline $77(24.7 \%)$ & $10-20$ & \\
\hline $99(31.7 \%)$ & $20-30$ & \\
\hline $58(18.03 \%)$ & $30-40$ & \\
\hline $36(11.60 \%)$ & $\leq 40$ & \\
\hline $25(8.06 \%)$ & Female & \multirow{2}{*}{ Gender } \\
\hline $275(91.70 \%)$ & Male & \\
\hline $58(19.1 \%)$ & Finger imputation & \multirow{8}{*}{ Cause of fracture } \\
\hline $154(50.8 \%)$ & Accident & \\
\hline $70(23.1 \%)$ & Being shot & \\
\hline $11(3.6 \%)$ & Falling & \\
\hline $3(0.7 \%)$ & Smashing & \\
\hline $7(2.3 \%)$ & Caught in door & \\
\hline $58(19.1 \%)$ & Finger imputation & \\
\hline $154(50.8 \%)$ & Accident & \\
\hline $297(97.04 \%)$ & No (immunized) & \multirow{2}{*}{ Needing tetabulin injection } \\
\hline $6(2.00 \%)$ & Yes (unimmunized) & \\
\hline $81(29.04 \%)$ & $>5$ & \multirow{5}{*}{ History of tetabulin injection } \\
\hline $36(11.88 \%)$ & $5-10$ & \\
\hline $61(20.13 \%)$ & $10>$ & \\
\hline $92(30.36 \%)$ & Not remembered & \\
\hline $33(10.89 \%)$ & Not vaccinated & \\
\hline $110(36.60 \%)$ & $10-15$ & \multirow{8}{*}{ Tetabulin injection duration } \\
\hline $48(15.08 \%)$ & 20 & \\
\hline $85(28.01 \%)$ & 30 & \\
\hline $9(3.09 \%)$ & 45 & \\
\hline $40(13.02 \%)$ & 60 & \\
\hline $5(1.70 \%)$ & 90 & \\
\hline $5(1.70 \%)$ & 120 & \\
\hline $110(36.60 \%)$ & $10-15$ & \\
\hline
\end{tabular}

$\checkmark$ The frequency is shown as number (percent) 
In this study, 300 patient cases with an open fracture referred to the ER of the Khatamolanbia Hospital in Zahedan in 2017 were assessed. Among 300 patients, 275 patients (91.7\%) were male and 25 patients (8.3\%) were female. The most frequent age range was 20 to 30 years old $(31.7 \%)$, and the least frequent ones were 5 to 10 years old $(10 \%)$ and more than 50 years old (11.6\%). Regarding tetabulin injection history: $29.04 \%$ of the patients injected tetabulin in less than 5 years, $11.88 \%$ injected it in 5 to 10 years, $20.13 \%$ injected it in more than 10 years, $30.36 \%$ didn't remember whether they had received it or not, and $10.89 \%$ had no history of tetabulin injection.

The most frequent cause of an open fracture among the patients in the ER of the Khatamolanbia hospital in Zahedan during 2017 was accident (50.8\%), and its lowest frequency was smashing (less than 1\%). The frequency of the unimmunized and immunized patients was $2 \%$ and $97.04 \%$ respectively. It means that according to the injection history and severity of the injury, $97 \%$ of the patients didn't need tetabulin injection and only $2 \%$ of them strongly needed tetabulin injection. The frequency of the patients based on their gender, age, cause of fracture, the need for tetabulin injection, and the history of tetabulin injection are listed in Table 1.

In order to determine the frequency of the patients with an open fracture in the Khatamolanbia hospital in 2017 who needed tetabulin injection based on their gender, Kappa Cohen's correlation coefficient (also known as Kappa coefficient correlation) was used. The results showed that the Kappa coefficient is $0.004(\mathrm{p}=0.339)$. Kappa coefficient ranges from 0 to 1 , and when it is closer to 1 , it implies more agreement. Kappa coefficient less than $0.4,0.4$ to 0.75 , and more than 0.75 imply the weak, good, and excellent agreement respectively. Therefore, the agreement between the frequency of the patients with an open fracture who needed tetabulin injection and their gender is 0.4 , and it is weak (Table 2).

Table 2. Determination of the frequency of the patients with an open fracture who needed tetabulin injection based on their gender using Kappa correlation coefficient

\begin{tabular}{llllll}
\hline Variable & & \multicolumn{1}{c}{ The need for tetabulin injection } & Kappa test & p \\
\hline \multirow{4}{*}{ Gender } & & Yes Frequency (percent) & No Frequency (percent) & & 0.399 \\
& Male & $5(83.33 \%)$ & $274(92.25 \%)$ & 0.004 & \\
& Female & $1(16.66 \%)$ & $23(7.74 \%)$ & & \\
& Total & 6 & 297 & & \\
\hline
\end{tabular}

To determine the frequency of the patients with an open fracture in the Khatamolanbia hospital in 2017 who needed tetabulin injection based on their age, Kappa Cohen's correlation coefficient (also known as Kappa coefficient correlation) was used. The results showed that the Kappa coefficient is 0.043 ( $\mathrm{p}=0.04)$. Kappa coefficient ranges from 0 to 1 , and when it is closer to 1, it implies more agreement. Kappa coefficient less than $0.4,0.4$ to 0.75 , and more than 0.75 imply the weak, good, and excellent agreement respectively. Therefore, the agreement between the frequency of the patients with an open fracture who needed tetabulin injection and their age is 0.4 to 0.75 , and it is good (Table 3). In the other words, as the age increases, the need for tetabulin injection for the patients with an open fracture also increases. This correlation is statistically significant $(\mathrm{p}=0.04)$.

Table 3. Determination of the frequency of the patients with an open fracture who needed tetabulin injection based on their age using Kappa correlation coefficient

\begin{tabular}{|c|c|c|c|c|c|}
\hline \multirow[t]{2}{*}{ Variable } & & \multicolumn{2}{|c|}{ Need for tetabulin injection } & \multirow[t]{2}{*}{ Kappa test } & \multirow[t]{2}{*}{$\mathbf{p}$} \\
\hline & & Yes Frequency (percent) & No Frequency (percent) & & \\
\hline \multirow{3}{*}{ Age } & $0-30$ & $4(66.66 \%)$ & $211(71.04 \%)$ & 0.043 & 0.04 \\
\hline & $>30$ & $2(33.33 \%)$ & $86(28.95 \%)$ & & \\
\hline & Total & 6 & 297 & & \\
\hline
\end{tabular}

To determine the frequency of the patients with an open fracture in the Khatamolanbia hospital in 2017 who received tetabulin based on their gender, Kappa Cohen's correlation coefficient (also known as Kappa coefficient correlation) was used. The results showed that the Kappa coefficient is $0.014(\mathrm{p}=0.780)$. Kappa coefficient less than 0.4 implies the weak agreement. Therefore, the agreement between the frequency of the patients with open fracture who received tetabulin and their gender is less than 0.4 , and it is weak (Table 4). 
Table 4. Determination of the frequency of the patients with an open fracture who received tetabulin based on their gender using Kappa correlation coefficient

\begin{tabular}{|c|c|c|c|c|c|}
\hline \multirow[t]{2}{*}{ Variable } & & \multicolumn{2}{|c|}{ Need for tetabulin injection } & \multirow[t]{2}{*}{ Kappa test } & \multirow[t]{2}{*}{ p } \\
\hline & & Yes Frequency (percent) & No Frequency (percent) & & \\
\hline \multirow{3}{*}{ Gender } & Male & $224(91.80 \%)$ & $55(93.22 \%)$ & 0.014 & 0.780 \\
\hline & Female & $20(8.19 \%)$ & $4(6.77 \%)$ & & \\
\hline & Total & 244 & 59 & & \\
\hline
\end{tabular}

Finally, in order to determine the frequency of the patients with an open fracture in the Khatamolanbia hospital in 2017 who received tetabulin based on their age, Kappa Cohen's correlation coefficient (also known as Kappa coefficient correlation) was used. The results showed that the Kappa coefficient is $0.046(\mathrm{p}=0.05)$. Kappa coefficient 0.4 to 0.75 implies the good agreement. Therefore, the agreement between the frequency of the patients with an open fracture who received tetabulin and their age is good (Table 5). In the other words, as the age increases, the frequency of tetabulin injection for the patients with an open fracture also increases. This correlation is statistically significant $(\mathrm{p}=0.05)$.

Table 5. Determination of the frequency of the patients with an open fracture who received tetabulin based on their age using Kappa correlation coefficient

\begin{tabular}{llllll}
\hline Variable & & & \multicolumn{1}{c}{ Need for tetabulin injection } & Kappa test & p \\
\hline \multirow{4}{*}{ Age } & & Yes Frequency (percent) & No Frequency (percent) & 0.046 & 0.05 \\
& $0-30$ & $171(70.08 \%)$ & $44(74.57 \%)$ & & \\
& $>30$ & $73(29.91 \%)$ & $15(25.42 \%)$ & & \\
\hline
\end{tabular}

\section{Discussion and Conclusion}

The goal of this study was to assess the consistency of tetabulin injection to the patients with an open fracture. This was a cross-sectional descriptive study. The population of the study was all the patients with an open fracture referred to the Khatamolanbia hospital in Zahedan in 2017. The statistical sample included 300 patients with an open fracture who were selected by random and available sampling. In this section of the study, the goal, research problem, and method of the study will be briefly discussed and conclusion and comparison will be made.

As mentioned in the previous section, the results showed that the Kappa coefficient for the frequency of the patients with an open fracture who needed tetabulin injection based on their gender is 0.004 . Kappa coefficient ranges from 0 to 1 , and when it is closer to 1 , it implies more agreement. Kappa coefficient less than 0.4, 0.4 to 0.75 , and more than 0.75 imply the weak, good, and excellent agreement respectively. Therefore, the agreement between the frequency of the patients with an open fracture who needed tetabulin injection and their gender is 0.4, and it is weak. In a study performed by Afzali et al. (2015) with the goal to assess the level of anti-tetanus antibody in the trauma patients referred to Shahid Beheshti hospital in Kashan, they realized that gender is not an effective factor on the level of anti-tetanus antibody in these patients (Afzali, Sharif, \& Mousavi, 2015). In their study, Nemati et al. (2014) also concluded that there was no significant relationship between the gender and anti-tetanus antibody level in the patients with type II diabetes (Nemati et al., 2014). In contrast, in a study performed by $\mathrm{Wu}$ et al. (2009) to assess the relationship between the decrease of tetanus antitoxin and age in Taiwan, they realized that the need for tetabulin injection was higher in men in comparison to the women. Therefore, they concluded that gender has a significant positive effect on the decrease of tetanus antitoxin in the serum (Wu, Ko, Lee, Tsai, Li, Pao, Lee, Chang, Shih, \& Ko, 2009). Also, in a study conducted by Kader et al. (2016) in Yazagat, Turkey, they concluded that gender has a significant positive effect on the decrease of tetanus antitoxin in the serum, and it is lower in men (Kader, Balci, \& Erbay, 2016).

As mentioned in the previous section, the results showed that the Kappa coefficient for the frequency of the patients with an open fracture who needed tetabulin injection based on their age is 0.043 . Kappa coefficient ranges from 0 to 1 , and when it is closer to 1 , it implies more agreement. Kappa coefficient less than 0.4, 0.4 to 0.75 , and more than 0.75 imply the weak, good, and excellent agreement respectively. Therefore, the agreement between the frequency of the patients with an open fracture who needed tetabulin injection and their age is 0.4 to 
0.75 , and it is good (table 3). In the other words, as the age increases, the need for tetabulin injection for the patients with an open fracture also increases. This correlation is statistically significant. Toker et al. (2017) assessed the immunity of the adult trauma patients against tetanus and concluded that as the age of the patients increases, their immunity against tetanus decreases. Therefore, the older trauma patients need tetabulin injection more than the younger ones (Toker et al., 2017). In their study, Mizuno et al. (2014) also concluded that there is a significant positive relationship between the age and decrease of anti-tetanus antibody level, which means as the age increases, the anti-tetanus antibody level in the serum decreases. This is more significant in the 50 years and older patients (Mizuno, Yamamoto, Takeshita, \& Takahashi, 2014).

According to the results obtained in this study, age is effective on the decrease of the serum level of anti-tetanus antibody. However, gender has no significant effect on the level of anti-tetanus antibody level. Thus, it can be concluded that the amount of tetabulin injection for open fractures is consistent with the national guideline.

\section{Conflict of interests}

The authors declare that there is no conflict of interests regarding the publication of this paper.

\section{References}

Afzali, H., Sharif, M. R., \& Mousavi, S. (2015). Determination of tetanus antibody levels in trauma patients referred to Shahid Beheshti Hospital in Kashan, Iran, 2014. Archives of Trauma Research, 4(3). https://doi.org/10.5812/atr.30687

Borella-Venturini et al. (2017). Tetanus vaccination, antibody persistence and decennial booster: A serosurvey of university students and at-risk workers. Epidemiology \& Infection, 145(9), 1757-62. https://doi.org/10.1017/S0950268817000516

Dong, M., Masuyer, G., \& Stenmark, P. (2019). Botulinum and tetanus neurotoxins. Annual Review of Biochemistry, 88, 811-37. https://doi.org/10.1146/annurev-biochem-013118-111654

Erdem, S., \& Blankson, M. A. (2013). Fractal-fracture analysis and characterization of impact-fractured surfaces in different types of concrete using digital image analysis and 3D nanomap laser profilometery. Construction and Building Materials, 40, 70-6. https://doi.org/10.1016/j.conbuildmat.2012.11.013

Finkelstein, P., Teisch, L., Allen, C. J., \& Ruiz, G. (2017). Tetanus: A potential public health threat in times of disaster. Prehospital and Disaster Medicine, 32(3), 339-42. https://doi.org/10.1017/S1049023X17000012

Graham, B. S., \& Ambrosino, D. M. (2015). History of passive antibody administration for prevention and treatment of infectious diseases. Current Opinion in HIV and AIDS, 10(3), 129. https://doi.org/10.1097/COH.0000000000000154

Jafari, N., Abolhasani, F., Naghavi, M., Pourmalek, F., \& Moradi, L. M. (2009). National burden of disease and study in Iran.

Kader, Ç., Balci, M., \& Erbay, A. (2016). Evaluation of tetanus antibody levels in adults in Yozgat, Turkey. Turkish Journal of Medical Sciences, 46(3), 646-50. https://doi.org/10.3906/sag-1503-38

Mizuno, Y., Yamamoto, A., Komiya, T., Takeshita, N., \& Takahashi, M. (2014). Seroprevalence of tetanus toxoid antibody and booster vaccination efficacy in Japanese travelers. Journal of Infection and Chemotherapy, 20(1), 35-7. https://doi.org/10.1016/j.jiac.2013.11.003

Mokhayeri et al. (2016). Burden of Vaccine-Preventable Diseases-Measles, Tetanus, Diphtheria and Whooping Cough-in Iran: Findings from the GBD study 2010. Archives of Iranian medicine.

Narang, M., Khurana, A., Gomber, S., \& Choudhary, N. (2014). Epidemiological trends of tetanus from East Delhi, India: A hospital-based study. Journal of Infection and Public Health, 7(2), 121-4. https://doi.org/10.1016/j.jiph.2013.07.006

Nemati et al. (2014). Lower serum level of anti-tetanus toxin antibodies in patients with type 2 diabetes mellitus. Acta Medica Indonesiana, 46(1).

O’Dwyer, L. (2017). Stiff as a board: Nursing the tetanus patient. BSAVA Congress Proceedings: BSAVA Library. https://doi.org/10.22233/9781910443439.39.5

Qadir, M. I., \& Komal, T. (2019). Public Awareness about Tetanus.

Toker et al. (2017). Tetanus immunity status among adult trauma patients in an ED. Turkish Journal of Emergency Medicine, 17(3), 95-8. https://doi.org/10.1016/j.tjem.2017.02.001 
Wu, C. J., Ko, H. C., Lee, H. C., Tsai, W. C., Li, M. G., Pao, Y. Z., Lee, N. Y., Chang, C. M., Shih, H. I., \& Ko, W. C. (2009). Decline of tetanus antitoxin level with age in taiwan. Journal of the Formosan Medical Association, 108(5), 395-401. https://doi.org/10.1016/S0929-6646(09)60083-8

Yen, C., Murray, E., Zipprich, J., Winter, K., \& Harriman, K. (2015). Missed opportunities for tetanus postexposure prophylaxis - California, January 2008-March 2014. MMWR Morbidity and mortality weekly report, 64(9), 243.

\section{Copyrights}

Copyright for this article is retained by the author(s), with first publication rights granted to the journal.

This is an open-access article distributed under the terms and conditions of the Creative Commons Attribution license (http://creativecommons.org/licenses/by/4.0/). 